\title{
Intelligent data analysis to interpret major risk factors for diabetic patients with and without ischemic stroke in a small population Fikret Gürgen*1 and Nurgül Gürgen ${ }^{2}$
}

\author{
Address: ${ }^{1}$ Dept of Computer Eng., Boaziçi University TR-80815 Bebek-Istanbul/TURKEY and ${ }^{2}$ Neurology Division, Lütfiye Nuri Burat Hospital \\ Sultançiftlii Istanbul/TURKEY \\ Email: Fikret Gürgen* - gurgen@boun.edu.tr; Nurgül Gürgen - gurgen@boun.edu.tr \\ * Corresponding author
}

Published: 4 March 2003

BioMedical Engineering OnLine 2003, 2:5
Received: 20 November 2002

Accepted: 4 March 2003

This article is available from: http://www.biomedical-engineering-online.com/content/2/l/5

(C) 2003 Gürgen and Gürgen; licensee BioMed Central Ltd. This is an Open Access article: verbatim copying and redistribution of this article are permitted in all media for any purpose, provided this notice is preserved along with the article's original URL.

\begin{abstract}
This study proposes an intelligent data analysis approach to investigate and interpret the distinctive factors of diabetes mellitus patients with and without ischemic (non-embolic type) stroke in a small population. The database consists of a total of 16 features collected from 44 diabetic patients. Features include age, gender, duration of diabetes, cholesterol, high density lipoprotein, triglyceride levels, neuropathy, nephropathy, retinopathy, peripheral vascular disease, myocardial infarction rate, glucose level, medication and blood pressure. Metric and non-metric features are distinguished. First, the mean and covariance of the data are estimated and the correlated components are observed. Second, major components are extracted by principal component analysis. Finally, as common examples of local and global classification approach, a k-nearest neighbor and a high-degree polynomial classifier such as multilayer perceptron are employed for classification with all the components and major components case. Macrovascular changes emerged as the principal distinctive factors of ischemic-stroke in diabetes mellitus. Microvascular changes were generally ineffective discriminators. Recommendations were made according to the rules of evidence-based medicine. Briefly, this case study, based on a small population, supports theories of stroke in diabetes mellitus patients and also concludes that the use of intelligent data analysis improves personalized preventive intervention.
\end{abstract}

\section{Introduction}

In modern medicine, large amounts of data are generated, but there is a widening gap between data collection and data comprehension. It is clear that a single human can not process all of the data available and make a rational decision of basic trends. Thus, there is a growing pressure for intelligent data analysis techniques to facilitate the creation of knowledge to support clinicians in making decisions [1-3].

Understanding the major risk factors of a diesease is an important factor for clinicians in prevention strategy. The attending physician plays an important role providing in- formation to reduce those risk factors. It is up to the physician whether to warn patients at risk about the major causes of a particular disease and the degree of risk that they are facing. Consider the example of a 66-year-old person who does not know about stroke (also in this study, ischemic stroke) but wants to know the risk of having certain medical test results outside of normal. Explaining the relative risk of stroke given the test results and given the evidence of previous cases interpreted with the aid of intelligent data analysis methods will make the situation clearer. 
Stroke is an important health issue worldwide and expressing and interpreting risk factors provides vital epidemiological information [4-13]. This study discusses a computational method for highlighting the major risk factors of a small population of diabetic patients with and without non-embolic stroke by performing dependency analysis with local and global classification aspects. For this purpose, the follow-up data of 22 diabetic patients with ischemic stroke (non-embolic) and 22 diabetic patients without stroke were collected over several years [6]. Average population age was $66.2 \pm 9.9$ For the stroke group, age was $66.2 \pm 9.9$ (mean and s.d.) years and $61 \pm$ 6.1 (mean and s.d.) years for the control group. Diabetes mellitus (DM) is diagnosed by a fasting glucose level higher than $140 \mathrm{mg} / \mathrm{dl}$ and random glucose levels higher than $200 \mathrm{mg} / \mathrm{dl}$ in repeated measurements. The study population of 44 patients was chosen with these glucose levels. Then, a set of tests were applied to construct the parameters of each feature vector. The tests include age, gender, duration of diabetes, cholesterol, high density lipoprotein (HDL), triglycerides levels, neuropathy, nephropathy, retinopathy, peripheral vascular disease, myocardial infarction rate, fasting and random glucose levels (FGL and RGL), medication, and systolic and diastolic blood pressures. The feature vectors thus contain both metric and nonmetric components. For example, a blood cholesterol level test is a metric component that can be processed with mathematical operations. On the other hand, retinopathy is a nonmetric component that provides a nominal scale to label or to identify retinal conditions.

This article discusses a limited number of cases of stroke in diabetic patients with primary risk factors for which preventive measures exist. Our purpose is to use existing knowledge for developing prevention strategies based on evidence-based medicine [13] that fit the patient and comply with current scientific concepts. Thus, this system transforms data into biomedical information that, together with expert knowledge, is helpful for decision making in patient care.

\section{A small population study: Diabetic patients with and without non-embolic stroke}

Using various diagnostic tests and searching for evidence of disease are generally routine for monitoring patients with diabetes mellitus (DM), and are also critical for patients $[4,5]$. Measuring major risk factors of ischemic stroke in patients with DM can also provide reasons for the attending physician to initiate preventive measures adapted to particular case. Typical microvascular complications are neuropathy, nephropathy, retinopathy; macrovascular complications are coronary artery diseases (CAD), and peripheral vascular diseases (PVD) [6,7]. Abnormal test results of cholesterol, HDL, triglycerides levels, FGL and RGL and sistolic and diastolic blood pressures are considered risk factors of nonembolicischemic stroke for DM patients [5-12]. Various studies have addressed the relationship between DM and stroke, and the probable risk factors [4-12]. Even though ischemic cerebravascular events are generally caused by macrovascular changes [7-9], in DM the cerebral ischemia is caused by small artery occlusion [6]. In DM, age, hypertension, MI, PVD are the known risks of stroke $[4,5]$ but few studies are available to indicate the relationship between neuropathy, nephropathy, retinopathy, and stroke.

Means, standard deviations and correlations are estimated from the observed population. It is known that small sample sizes reduce the statistical power of the study and generally result in wide confidence interval of estimated parameters. Thus, identical studies on larger samples may identify more important differences that have gone undetected here. Also, deviation from the normal may cause errors in parameter estimations. As compensation for the small size of the study population, we first observe the statistics with an assumption of normal distribution, then attempt to employ nonparametric classification techniques such as k-NN and MLP with the jackknife method. Although there exist ways of checking the fit: for example, how well the normality of distribution for each component can be evaluated by various means such as the Kolmogorov-Smirnov test, our aim is to build a decision support mechanism. Regardless of the limitations imposed by technical artifacts and sample size constraints, our results describe several important findings that emerged from this preliminary investigation.

Mean and standard deviation of metric components: cholesterol, HDL, triglycerides levels, FGL and RGL, and systolic and diastolic blood pressures are presented in Table 1. Correlation estimates of pairwise relationships of the components were calculated, as presented in Tables 3 and 4 . Nonmetric components were counted as the percentage of existing or nonexisting cases, as given in Table 2.

\section{Investigating principal factors of data}

Among the factor analysis methods [13-16], principal component analysis (PCA) is used to obtain independent factors from data. PCA is based on obtaining eigenvalues and eigenvectors from the covariance matrix of data. The covariance matrix is transformed into a diagonal matrix by applying an orthogonal transformation, with the factors arranged in relative order of importance. The estimation of the covariance matrix, $\hat{\Sigma}$ is

$$
\hat{\Sigma}=\frac{1}{n} \sum(x-\mu)(x-\mu)^{T}
$$


Table I: Statistics of metric components of DM patients with and without ischemic stroke

\begin{tabular}{lcccc}
\hline & \multicolumn{2}{c}{ Ischemic stroke } & & No stroke \\
\cline { 2 - 5 } & Mean & St. Dev. & Mean & St. Dev. \\
\hline Age & 66.2 & 9.9 & 61 & 6.1 \\
Cholesterol & 203.4 & 54.5 & 217.8 & 50.9 \\
Triglyceride & 150.5 & 97.8 & 1556.0 & 89.9 \\
HDL & 41.0 & 6.5 & 44.1 & 6.8 \\
RGL & 206.6 & 86.2 & 196.2 & 53.0 \\
FGL & 187.3 & 75.3 & 180.3 & 62.5 \\
Systolic & 143.6 & 26.3 & 143.2 & 14.6 \\
Diastolic & 83.6 & 12.9 & 85 & 11.0 \\
DM (month) & 97.9 & 91.1 & 186.3 & 87.7 \\
\hline
\end{tabular}

Table 2: Statistics of non-metric components of DM patients with and without ischemic stroke

\begin{tabular}{|c|c|c|}
\hline & first class & second class \\
\hline \multicolumn{3}{|l|}{ Gender } \\
\hline Female & $6(\% 27.3)$ & $8(\% 36.4)$ \\
\hline Male & $16(\% 72.7)$ & $14(\% 63.6)$ \\
\hline \multicolumn{3}{|l|}{ Medicine } \\
\hline Used & II (\%50) & 17(\%77.3) \\
\hline Not used & II (\%50) & $5(\% 22.7)$ \\
\hline \multicolumn{3}{|l|}{ Neuropathy } \\
\hline Exist & $15(\% 68.2)$ & $19(\% 86.3)$ \\
\hline Nonexist & $7(\% 31.8)$ & $3(\% \mid 3.7)$ \\
\hline \multicolumn{3}{|l|}{ Nephropathy } \\
\hline Exist & $7(\% 31.8)$ & $9(\% 40.9)$ \\
\hline Nonexist & $15(\% 68.2)$ & $13(\% 59.1)$ \\
\hline \multicolumn{3}{|l|}{ PVD } \\
\hline Exist & $6(\% 27.3)$ & $7(\% 31.8)$ \\
\hline Nonexist & $16(\% 72.7)$ & $15(\% 68.2)$ \\
\hline \multicolumn{3}{|l|}{ Retinopathy } \\
\hline Exist & $4(\% \mid 8.2)$ & $2(\% 9.1)$ \\
\hline Nonexist & $18(\% 81.8)$ & $20(\% 90.9)$ \\
\hline \multicolumn{3}{|l|}{ MI } \\
\hline Exist & $4(\% \mid 8.2)$ & $\mathrm{I}(\% 4.5)$ \\
\hline Nonexist & $18(\% 81.8)$ & $21(\% 95.5)$ \\
\hline
\end{tabular}

Here, the $x_{I}$ 's are constituent vectors for sample space, and $\hat{\mu}$ is the mean value. PCA compresses information to eigenvalues, $\lambda_{1}, \lambda_{2}, \ldots, \lambda_{n}$ and eigenvectors, $\mathrm{u}_{1}, \mathrm{u}_{2}, \ldots, \mathrm{u}_{\mathrm{n}}$ of $\hat{\Sigma}$. The matrix $\mathrm{x}^{\mathrm{T}} \mathrm{u}_{\mathrm{i}}$ and the eigenvectors $\mathrm{u}_{\mathrm{i}}, \mathrm{i}=1, \ldots, \mathrm{p}$ are needed for recovering data. The reconstruction error $\mathrm{R}^{2}$ is due to the neglecting small eigenvalues:

$$
R^{2}=\operatorname{trace}[D]-\operatorname{trace}\left[D_{M}\right]=\sum_{n=M+1}^{N} \lambda_{n}
$$

where $\mathrm{D}$ is the diagonal eigenvalue matrix and $\mathrm{R}^{2}$ is the error arising from the eigenvalues $\lambda_{n^{\prime}} \mathrm{i}=\mathrm{M}+1, \ldots \mathrm{N}$ that are left out.

By PCA analysis of all components, we find the first macrovascular components of Table 1: cholestrol, trigliseride, glucose, and blood pressures to be the principal components. From a decision making point of view, we observe that these components are the most informative with regard to in the population study. In fact, this finding confirms the reports of major risk factors reported in the literature [4-13]. Among the other components, age and 
Table 3: Results of k-NN method

\begin{tabular}{llll}
\hline & All Components & Principle Components \\
\cline { 3 - 4 } & & & \% for total \\
$\mathrm{n}=1$ & 52.23 & 52.3 \\
$\mathrm{k}=3$ & 65.9 & 65.9 \\
$\mathrm{k}=5$ & 68.2 & 68.2 \\
$\mathrm{k}=7$ & 63.6 & 63.6 \\
$k=10$ & 68.2 & 68.2 \\
$k=20$ & 63.6 & 63.6 \\
$\mathrm{k}=40$ & 29.5 & 29.5 \\
\hline
\end{tabular}

$\begin{array}{ll}5 & 66 \\ 7 & 65\end{array}$

gender are designated during data collection and have less information. Microvascular changes, neuropathy, nephropathy, retinopathy, are also assessed by only two distinctive levels (existence-nonexistence) to show the subgroups of DM subjects. Thus, they too have limited information that can be used for decision making. PCA is found to be valuable for summarizing the trends of features of high-risk patients with DM.

\section{Decision making by principal factors}

It is known that there is no standard approach to establish the optimum decision criteria for a generic problem. Nonparametric classification approaches [19-22] overcome the difficulty of accurate estimation of underlying distributions from small population size. These approaches are used without the assumptions about the form of the density function. Their decision procedures bypass probability estimation and go directly to decision functions. Due to the small population, we here employ nonparametric techniques instead of parametric ones such as K-Means, vector quantization (VQ) and gaussian mixture model (GMM) which, otherwise, are very useful with the large population. Fuzzy modeling methods could be used to also enhance the decision ability [23].

Among various nonparametric techniques, we choose a local k-NN and a global polynomial classifier, MLP. The concept of locality and globality is related to the location of information that is extracted for class decision. The kNN rule employs local information, in contrast, an MLP extracts global information.

It is known that the nearest neighbor in the feature space gives the half of the classification information obtained by the Bayes classifier [19]. On the other hand, polynomials have excellent properties as classifiers. Thus, polynomial classifiers are universal approximators relative to the optimal Bayes classifier [20]. Typical polynomial classifiers have either been based on either statistical methods or minimizing a mean-squared error criterion. The focus has been on linear and second-degree polynomial classifiers, but both of these methods traditionally have had limitations. Classical statistical methods estimate the mean and covariance for a parametric model [19-22]. These methods with a large number of features or multivariables lead to large intractable problems $[17,18]$.

With the k-NN rule, the class decision of an unknown sample is based on the majority of the nearest $\mathrm{k}$ samples. In other words, a local voting process decides the class of the unknown by the highest vote. This is, in fact, an approximation of Bayes Decision Theory in a local environment [19]. This process can further be extended to weighted voting and gaussian weighted voting (Parzen window). To recognize pattern $\mathrm{v}, \mathrm{k}$ minimum distance samples are computed among all the samples. The dis- 


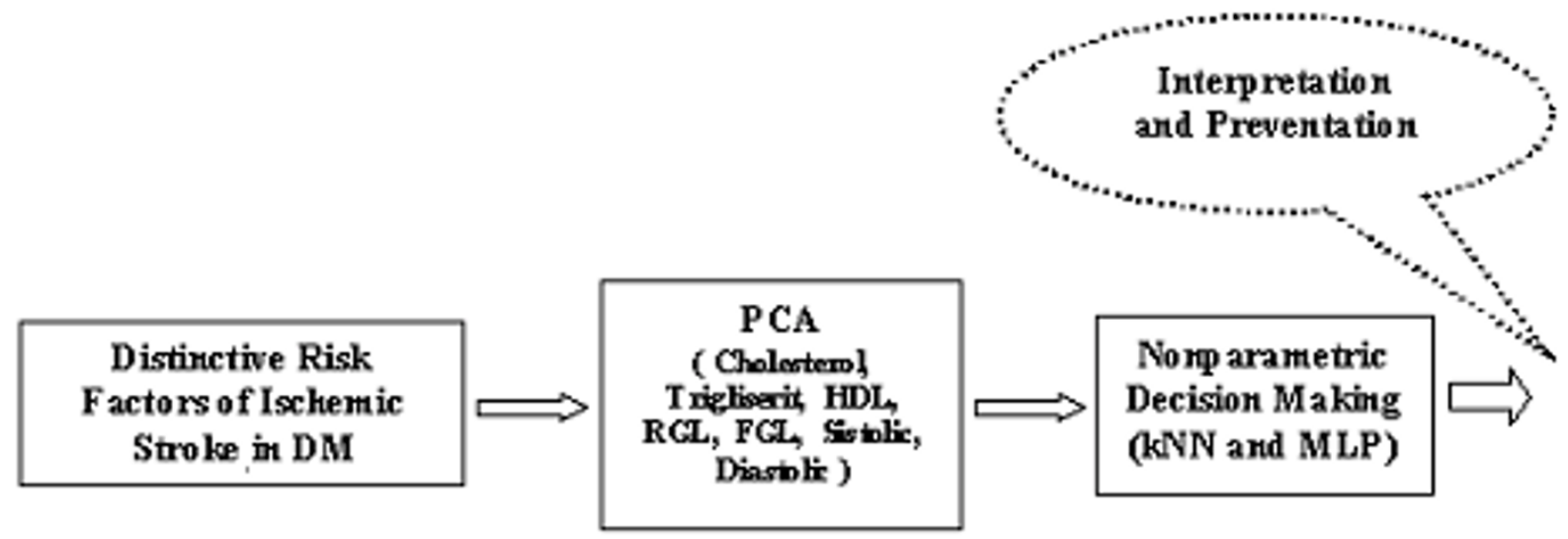

Figure I

Distinctive risk factors of DM patients with and without stroke

tance can be computed by various norms: minkowskinorm-based distances, e.g. euclidian as second order, covariance-based distance or mahalonobis, entropybased distance or kullback-leibler distances. The simplest and the most suitable process for a small sample size is the euclidian metric, which is defined as

$d j=\left|v-v_{j}\right|^{2}=\left(v-v_{j}\right)^{T}\left(v-v_{j}\right)$

where dj defines the distance between patterns $v$ and $v_{j}$. The k-NN rule classifies $\mathrm{v}$ by assigning it a label that is the most frequently represented among the k nearest samples:

$\mathrm{ki}=\max \left\{\mathrm{k}_{1}, \ldots, \mathrm{k}_{\mathrm{L}}\right\} \mathrm{x} \in \mathrm{w}_{i}$

$\mathrm{k}_{1}+\ldots+\mathrm{k}_{\mathrm{L}}=\mathrm{k}$

Here, $\mathrm{k}_{\mathrm{i}}$ is the number of neighbors belong to class $\mathrm{w}_{\mathrm{i}}(\mathrm{i}=$ $1, \ldots . \mathrm{L})$ class among the $\mathrm{k}$ nearest neighbors.

The global MLP [22] is a parallel, feedforward structure that consists of input, output, and hidden layers. Each layer has sigmoidal units interconnected through weight connections. The MLP is trained with a supervised back propagation (BP) algorithm to efficiently compute partial derivatives of an approximating function $\mathrm{F}(\mathrm{w} ; \mathrm{x})$. The network has an adjustable weight vector, w, that is computed with respect to all training data for a given value of input vector $\mathrm{x}$ and output vector $\mathrm{y}$. The weights are adjusted to fit a set of surfaces to the input space. The surfaces are con- structed with sigmoids by using the best linear regression concerning the cluster membership. The mean square (MSE) (Eq. 5) error function, which is the difference between the network's output and the supervisor output, is minimized to find the cluster membership:

$$
M S E=\sum_{q}\left(y_{q}-F\left(w ; x_{q}\right)\right)^{2}
$$

In k-NN rule, an arbitrary discriminant function is constructed for class decision. The nearest neighbor contributes the half of the classification information. An MLP can generate any nonlinear discriminant function of input by incorporating multiple constraints. Each sigmoidal unit contributes to the global discriminant function by a linear constraint.

\section{Results}

The study population contains data relating to 44 diabetic patients' collected by neurology specialists. The data have a total of 16 metric and non-metric components. The computer-based system uses two stages: first, the PCA method reduces the features to seven dimensions. The best discriminators for class membership are then searched by nonparametric classifiers, k-NN and MLP. Multi-parameter classifiers were found to significantly improve upon the classification performance of single parameter designs. Instead of single parameter based 


\section{Trigliserit -RGL-cholesterol data stroke( $x)$ vs nonstroke(o)}

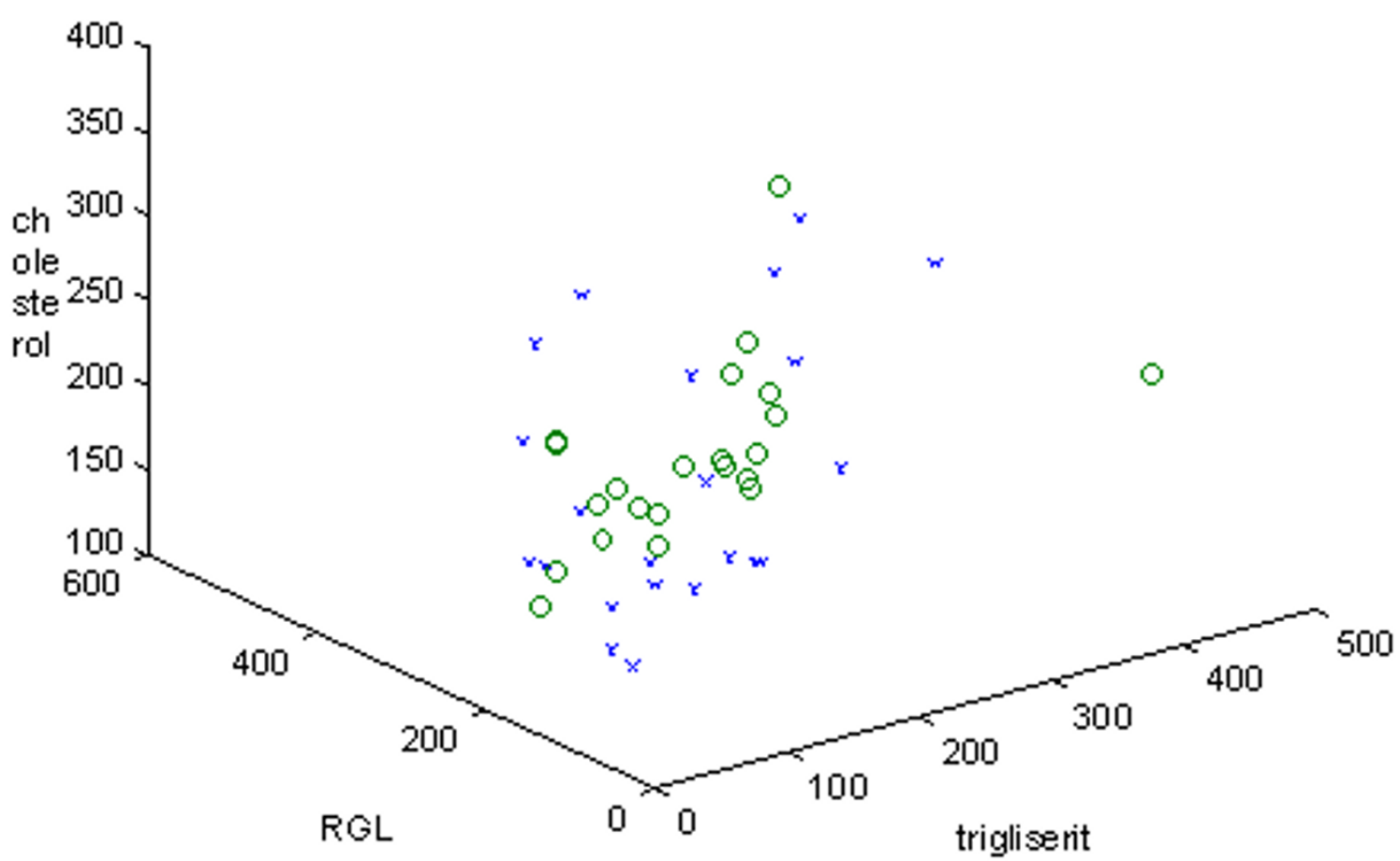

Figure 2

Visual information of triglyceride-RGL-cholestrol

conclusions, we employed the decision produced by major risk factors. The method gives the study stronger arguments on the distintive factors. The small sample population size has an acknowledgable potential effect on the statistical power of the study, but we use classification techniques like k-NN and MLP to help overcome this drawback.

The results are shown in Table 3 and Table 4 . In case of k$\mathrm{NN}$, training and test sample sets are equivalent. An average classification score of $68.2 \%$ is obtained for k-NN. But in the case of MLP, we implement an hold-n-out method (jacknife, cross-validation) which is proven to be the most common method of network construction and validation, train-and-test, in cases where data are scarce. Test results are repeated with different sets using 10 test datum points and the remaining data for training. The average performance of five MLP experiments is found to be $66 \%$. Both classifiers identify the macrovascular changes as the best discriminators for this case study of stroke.

Figure 2 shows the component clusters and clearly provides a convincing picture to the physician and patients as the end-users. This easy-to-use graphic offers users information about the limits of the major risk factors.

\section{Discussions and Conclusion}

The aim of this study is to support physicians with intelligent data analysis techniques by providing what may be thought of as an indirect assistance tool. This paper presents a computer-based decision support system for physicians to improve prevention measures for ischemic stroke in DM patients. PCA helps to identify the basic trends that distinguish two cases: ischemic stroke and no stroke in DM. In this study, major distinctive factors are found to be cholesterol, triglycerides level, fasting and random glucose levels (FGL and RGL), and systolic and 
diastolic blood pressures. Physicians, furthermore, can suggest dietary supplements, drugs, exercises, etc. for the prevention of ischemic stroke for diabetic patients. Graphics of the components also support the discussion with clues for prevention.

We also plan to improve the information of diagnosis with a certain risk assessment factor. A grading for the risk of ischemic stroke for diabetic patients will support the prevention measures according to evidence-based medicine.

The major concern of the study is that the investigators could not have a bigger size of study population. This raises concerns about the meaningfulness of results but the authors believe that the methods used here clearly useful to overcome this handicap. Also the results of the study are supported by the risk factor findings of the other stroke and diabet studies in the literature [4-13]. When there is a large database, this will provide more convincing statistical power to the study. In this case, the general statistics will confirm the results and parametric decision making techniques such as expectation-maximization (EM) algorithm would be utilized.

The other limitation is the inability to directly account for certain microvascular factors in the decision process due to imprecision of their records: for example, retinopathy has only two identifying labels as exist or non exist. In fact, there are typically four cases that can be distinguished by the physician but the limited data size prevents us to include finer details. This may cause an error. The lack of precision, in general, reduces the effect of the feature in decision making.

Among the various other tests, we also believe that the doppler ultrasound measurements may present more detailed information on this case study. The areas of arteries and veins can be monitored for deciding the stages of the disease, and the condition of occlusions can be important for the preventive follow up.

The other weak point of this investigation may be the quality issues of the recording such as the use of nonstandardized procedures, the use of potentially low grade equipment or the measurement quality. However, as best supported by a group of physicians' observations, this was not the case.

As a conclusion, this case study points at a friutful line of enquiry in intelligent medical data analysis and the content of the work can further be extended to the other areas of stroke diagnosis and prevention.

\section{Acknowledgement}

We would like to express our thanks to Boaziçi University BAP 03 A 103 for their support. We would also thank to Editor of Biomedical Engineering Online Prof. Alwin Wald.

\section{References}

I. Brause and Rudiger Medical Data Analysis. Springer-Verlag 2000,

2. Hand DJ, Kok JN and Bertholt MR Advances in Intelligent Data Analysis. Springer-Verlag 1999, 89-93

3. Gurgen F Neural-Network-Based Decision Making in Diagnostic Applications. IEEE Engineering in Medicine and Biology 1999, 89-93

4. Barnett HJM, Mohr JP, Stein BM and Yatsu FM Stroke: Pathophysiology, Diagnosis and Management. Secon Edition, Churchill Livingstone Inc 1992,

5. Bogousslavssky J and Caplan L Stroke Syndromes. Cambridge University Press 1995,

6. Aydýn N, Esgin H, Yilmaz A, Gözeten F and Utku U Diabetes Mellitus'lu Non-Embolik Stroklu Olgularda Retinopati ve Dier Risk Faktörleri. In Proceeding of theTürk Beyin Damar Hastalýklarý Dernei 3. Sempozyumu 1999 ,

7. Silvestrini M, Rizzato B, Placidi F, Baruffaldi R, Bianconi A and Diomedi M UKPDS 60: Risk of Stroke in Type 2 Diabetes Estimated by the UK Prospective Diabetes Study Risk Engine. Stroke 2002, 33: $1776-|78|$

8. Horenstein RB, Smith DE and Mosca L Cholesterol Predicts Stroke Mortality in the Women's Pooling Project. Stroke 2002, 33:1863-1868

9. Muntner P, Garrett E, Klag MJ and Coresh J Trends in Stroke Prevalence Between 1973 and 199 I in the US Population 25 to 74 Years of Age. Stroke 2002, 33:1209-1213

10. Petty GW, Brown RD Jr, Whisnant JP, Sicks JD, O'Fallon WM and Wiebers DO Ischemic Stroke Subtypes: A Population-Based Study of Functional Outcome, Survival, and Recurrence. Stroke 2000, 31:1062-1068

II. David SH and Bell MB Stroke in the diabetic patient. Diabetes Care. Stroke 1994, 17:213-219

12. Jose B and Love BB Diabetes and stroke. Medical Clinics of North America 1993, 77(1):95-109

13. Diana BP and Hilarey B Retinopathy as a Risk Factor for Nonembolic Stroke in Diabetic Subjects. Stroke 1995, 26:593-596

14. Abbott RD and Donahue RP Diabetes and Risk of Stroke. JAMA 1987, 257:949-952

15. Mortel KF, Meyer JS, Sims PA and McClintic K Diabetes Mellitus as a Risk Factor for Stroke. Southern Medical Journal 83:904-9I I

16. Sackett DL, Rosenberg WM, Gray JA, Haynes RB and Richardson WS Evidence-based Medicine: What it is and what it isn't. Mr Med J 1996, 312:71-72

17. Sharma S Applied Multivariate Techniques. John Wiley 1996,

18. Rencher AC Methods of Multivariate Analysis. John Wiley 1995,

19. Duda RO and Hart PE Pattern Classification and Scene Analysis. 1972,

20. Devroye L, Gyorfi L and Lugosi G A Probabilistic Theory of Pattern Recognition. NewYork: Springer Verlag 1996,

21. Fukunaga K Introduction to Statiscal Pattern Recognition. Academic Press 1990,

22. Schürmann J Pattern Classification, A Unified View of Statistical and Neural Approaches. John Wiley \& Sons Inc 1996,

23. Takagi $T$ and Sugeno $M$ Fuzzy identification of systems and its applications to modelling and control. IEEE Trans. Systems Man and Cybernetics I985, I 5(1): I I6-132 